\title{
Votation générale 2007 de FMH Services
}

\section{Dr Jacques de Haller, président de FMH Services}

Reinhard Kunz, directeur

Correspondance:

FMH Services

Thorackerstrasse 3

Case postale

CH-3074 Muri b. Bern

Tél. 0419250077

Fax 0419250586

mail@fmhservices.ch
La votation générale 2007 représente une étape importante dans l'histoire de FMH Services. En y participant, vous entérinerez un processus de restructuration qui aura duré plus de deux ans et accepterez notre réorganisation en une entreprise autonome proposant ses services aux membres de la FMH. Notre firme est solide, financièrement saine et accumule les succès. Ses structures ont été allégées, ses prestations et ses produits sont unanimement reconnus.

Avec la convention-cadre passée entre la FMH et FMH Services, nous avons redéfini les relations entre les deux partenaires et posé des bases claires concernant nos orientations et notre gestion. Nos prestations sont destinées aux médecins libres praticiens. Notre objectif est de devenir encore plus opérationnels et de nous rapprocher de vous. A cet effet, nous travaillerons avec un nouveau conseil d'administration, que nous vous proposons d'élire dans la composition suivante et dont nous avons le plaisir de vous présenter les membres sous la forme de brefs portraits:

- Dr Donatella Campus Souche, déléguée de la SMSR (nouvelle);
- M. Sven Bradke, entrepreneur (nouveau);

- Dr Max Giger, délégué du CC FMH (ancien);

- Dr Vincenzo Liguori, délégué de l'OMCT (nouveau);

- Dr Thomas Paly, délégué du VEDAG (nouveau);

- Dr Max Giger, président de FMH Services (nouveau).

La nouvelle forme d'organisation et de direction définie en commun nous permettra de défendre les intérêts du corps médical en fonction de la politique de la FMH et de nos principes communs. Les nouveaux statuts de FMH Services reflètent cette orientation et nous donnent les moyens de collaborer efficacement.

Conjointement avec l'approbation du rapport d'activité et des comptes 2006, l'octroi de la décharge au conseil d'administration et la nomination de l'organe de contrôle, nous vous prions de donner votre voix aux membres du nouveau conseil d'administration et d'accepter les nouveaux statuts de FMH Services. Nous vous remercions d'ores et déjà de participer nombreux à la votation générale. 